\title{
Evaporation and contraction of a droplet that wets a surface monitored by photoacoustic detection
}

\author{
L. C. M. Miranda \\ Laboratório Associado de Sensores e Materiais, Instituto Nacional de Pesquisas Espaciais, \\ Caixa Postal 515, 12201 São José dos Campos, São Paulo, Brazil \\ N. Cella \\ Instituto Politécnico do Rio de Janeiro, Caixa Postal 97282, 28630 Nova Friburgo, Rio de Janeiro, Brazil
}

(Received 30 March 1992)

\begin{abstract}
The evaporation and contraction of a droplet wetting a flat metallic surface is monitored using photoacoustic detection. The results are interpreted in terms of an effective backing model together with the lubrication theory for droplet dynamics.
\end{abstract}

\section{INTRODUCTION}

Photoacoustic (PA) detection has proved over the past 15 years to be a reliable and useful technique for the investigation of the optical and thermal properties of liquids, gases, and solid materials. This versatility of PA detection is essentially due to the fact that PA detection consists basically of the measurement of the heat produced in a sample due to the absorption of a modulated radiation beam. The absorption of a radiation beam and the subsequent nonradiative deexcitation processes give rise to a heat source in the sample, which may be distributed throughout its volume or confined to its surface. This heat source generates both temperature and pressure fluctuations within the sample, which are then detected by thermal or acoustic, or even both, sensing devices. The heating of the sample by the incident beam depends not only on the amount of heat produced in the sample (i.e., on the sample absorption coefficient for the incident radiation beam as well as on the light-into-heat conversion efficiency) but also on how this heat diffuses through the sample. The dependence of the PA signal on the absorption coefficient for the incident radiation allows us to perform spectroscopic studies, whereas the fact that the signal is also proportional to the light-into-heat conversion efficiency means that it is complementary to other protoinduced energy conversion processes. This last aspect allows us to get information concerning the nonthermal deexcitation process occurring within the sample. The PA signal dependence on the heat diffusion through the sample allows us to perform not only the sample thermal characterization (i.e., measurements of its thermal diffusivity and conductivity) but also to conduct thermal imaging. This last point comes from the fact that the thermal wave generated by the absorption of an incident beam may be reflected and scattered as it encounters cracks, defects, and so on, thereby affecting the detected signal. We refer to Refs. 1 and 2 for a more detailed description of the PA effect.

More recently, the PA technique has been applied to study several transient phenomena, such as, laser-induced polymer ablation, ${ }^{3,4}$ oxygen evolution during photosynthesis, ${ }^{5}$ and gas adsorption and desorption in porous sub- stances. $^{6}$ In this paper we apply PA detection to the investigation of another type of transient phenomena, namely, the spreading and contraction of a solvent droplet that wets a surface. The purely fluid dynamics problem of the movement of small fluid droplets on a flat surface has gained renewed interest not only for its application to the technologically important problem of adhesion, but also for its analogous biological problem of cell motion. ${ }^{7,8}$ For a review on the spreading of liquid drops on solids we refer to the work of Rosenblat and Davis. ${ }^{9}$

\section{EXPERIMENT}

The PA investigation of liquid droplet motion was carried out using the experimental setup shown in Fig. 1. It consists basically of using the same type of PA cell configuration as that previously used in studying adhesive curing $^{10}$ and in thermal diffusivity measurements. ${ }^{11,12} \mathrm{~A}$ chopped 10-mW He-Ne laser beam, after being expanded, is shone on a $14-\mathrm{mm}$-diam Al disk, $12 \pm 1 \mu \mathrm{m}$ thick. This Al foil is closing one of the 8-mm-diam openings of a 2mm-long cylindrical brass cell cavity. The other cavity opening is closed with a BK-7 glass window. The laser

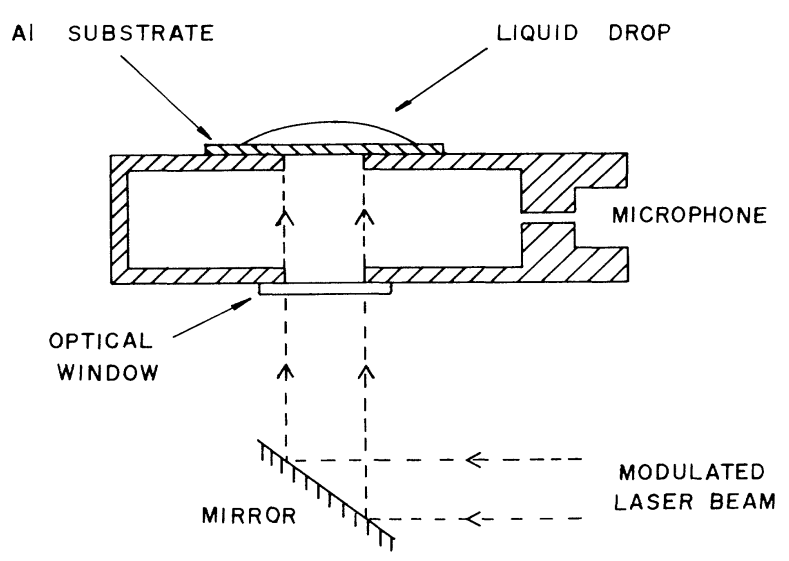

FIG. 1. Schematic arrangement of our experimental PA setup. 
beam was expanded to ensure uniform light absorption by the Al substrate. An electret microphone, coupled to the PA cell cavity through a $1-\mathrm{mm}$-diam duct located in the cavity wall, is used to sense the pressure fluctuations in the PA cavity produced by the periodic heating of the substrate due to the pumping beam. The microphone signal is fed into a lock-in amplifier, whose output signal is then recorded as a function of time. The droplets were deposited in the outer surface of the Al substrate with the help of a syringe. Before positioning the Al substrate in the PA cell, it was degreased by immersion in heated carbon tetrachloride followed by ultrasound cleaning. The liquids used were chloroform, carbon tetrachloride, trichloroethylene, and isopropanol. One drop of the first three solvents used, after the initial substrate wetting, covered essentially the whole sensing area, i.e., their initial diameters were of the order of $9 \pm 1 \mathrm{~mm}$. The isopropanol drop, in contrast, wetted the entire Al substrate so that its initial diameter was roughly $14 \mathrm{~mm}$. To check the reproducibility of our results, we have carried out a series of four to five experimental runs for each substance. After each measurement the Al substrate was carefully cleaned with $\mathrm{CCl}_{4}$ using cotton-wool sticks.

In Figs. 2 and 3 we show the time-dependent PA signal for one drop of chloroform and isopropanol recorded at a $160-\mathrm{Hz}$ modulation frequency, respectively. For both carbon tetrachloride and trichloroethylene, the PA signal exhibited the same behavior as that of the chloroform drop, except for the obvious change in the time length of the experiment. The first aspect to be noted in Figs. 2 and 3 is the decrease of the PA signal following the drop deposition and spreading. This signal decrease is explained as follows. Before the liquid drop deposition, the substrate has a high thermal impedance which minimizes its heat exchange with the surrounding air. After drop deposition and spreading, the liquid layer enhances the heat exchange so that the net substrate temperature rise is smaller than without the liquid layer. Furthermore, part of the substrate heat is extracted by the thin liquid layer as evaporation heat, which further decreases the PA signal.

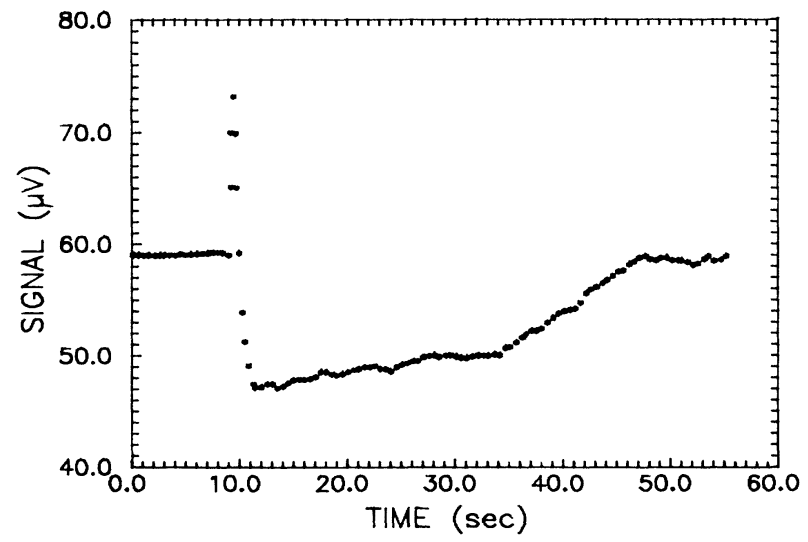

FIG. 2. Time dependence of the PA signal for a chloroform droplet recorded at a $160-\mathrm{Hz}$ modulation frequency.

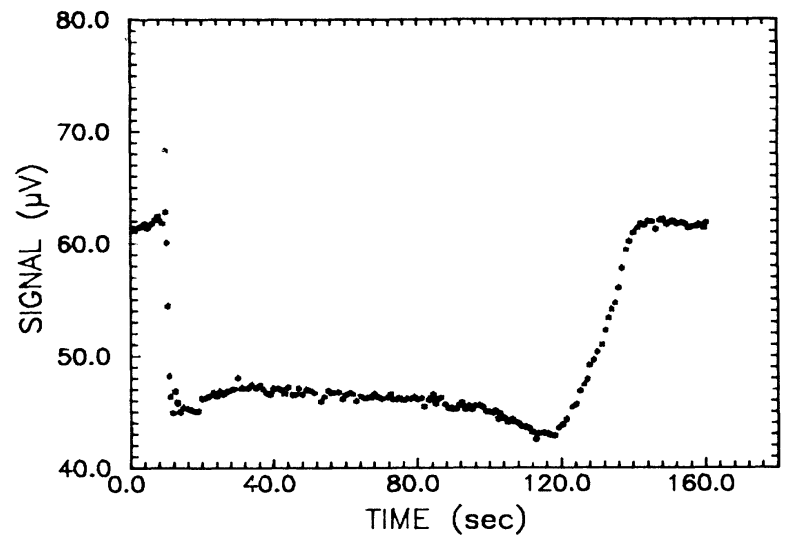

FIG. 3. Time dependence of the PA signal for an isopropanol droplet recorded at a $160-\mathrm{Hz}$ modulation frequency.

For chloroform and the other solvent droplets, following this initial decrease, the PA signal increases slowly as time elapses, until it reaches a region of fast increase. In contrast, for the alcohol droplet, the PA signal remains roughly constant, then goes through a minimum which is followed by fast rising. These differences between the solvent and the isopropanol droplets behavior are further confirmed by visual inspection of the droplets motion during the recording period. It was observed for the solvent droplets that, after the initial spreading, the droplet begins to contract its radius at a constant rate until it reaches a point where its diameter is roughly half of its original size. At this point, the drop motion slows down and it seems to almost "stop" contracting. Just after this point the drop begins to contract again but at a faster rate. The first regime up to the point where the droplet seems to stop contracting corresponds in Fig. 2 to the initial region of monotonic slow increase, whereas the fast contracting regime corresponds to the fast rising signal region in Fig. 2. For isopropanol, the drop dynamics is markedly different. In this case, the drop evaporates without changing its radius. In the next section, we present a model for the time-dependent PA signal describing quantitatively the drop motion and evaporation.

\section{THEORETICAL MODEL}

Consider the geometry shown in Fig. 4(a) representing schematically the physical situation of our experiments. A modulated light beam $I_{0} \exp (j \omega t)$ is incident on the substrate $(s)$ of thickness $l_{s}$ which is in contact with the air $(g)$ of the PA cavity and the interfacial layer $(e)$ of thickness $l$. We denote by $\alpha_{s}, e_{s}$, and $k_{s}$ the thermal diffusivity, effusivity, and conductivity of the substrate and by $\alpha, e$, and $k$ those of the effective interfacial layer. According to the thermal diffusion model of Rosencwaig and Gersho, ${ }^{13}$ the PA signal is determined by the temperature fluctuation $\Theta$ at the air substrate at $x=0$. Solving the thermal diffusion equation for the geometry shown in Fig. 4(a), one gets 

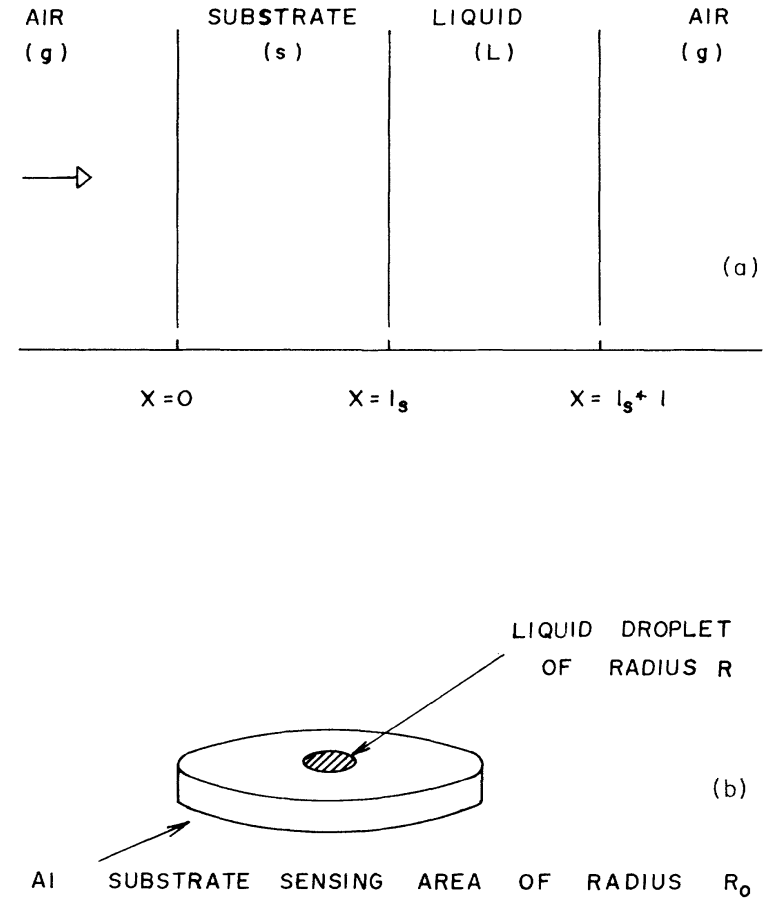

FIG. 4. (a) Schematic geometry for the PA cell. (b) Partial coverage of the substrate sensing area by the liquid droplet at some time after the droplet deposition.

$$
\Theta=\frac{\beta^{\prime} I_{0}(1+\bar{b}) e^{l_{s} \sigma_{s}}+(1-\bar{b}) e^{-l_{s} \sigma_{s}}}{k_{s} \sigma_{s}(1+\bar{b}) e^{l_{s} \sigma_{s}}-(1-\bar{b}) e^{-l_{s} \sigma_{s}}},
$$

where $\quad \bar{b}=b \tanh (l \sigma)$. Here $\sigma_{s}=(1+j) a_{s}$ and $\sigma=(1+j) a$ are the complex thermal diffusion coefficients of the substrate and the interfacial layer, respectively, $a_{s}=\left(\pi f / \alpha_{s}\right)^{1 / 2}, a=(\pi f / \alpha)^{1 / 2}, b=e / e_{s}$ is the ratio of the interfacial layer to the substrate thermal effusivities, and $\beta^{\prime}$ is the substrate surface absorption coefficient. In arriving at Eq. (1), we have assumed that the substrate is optically opaque and that the heat flux into the surrounding air is negligible. In the absence of the liquid layer, Eq. (1) reduces to the well-known expression

$$
\Theta_{0}=\frac{\beta^{\prime} I_{0}}{k_{s} \sigma_{s}} \frac{\cosh \left(l_{s} \sigma_{s}\right)}{\sinh \left(l_{s} \sigma_{s}\right)} \text {. }
$$

For the case of a thermally thin substrate (i.e., $\left.l_{s} \sigma_{s} \ll 1\right)$, which is quite adequate for our case of a 12$\mu \mathrm{m}$-thick $\mathrm{Al}$ foil at modulation frequencies below $1 \mathrm{kHz}$, using Eqs. (1) and (2), the ratio $\Theta / \Theta_{0}$ reduces to

$$
\frac{\Theta}{\Theta_{0}}=\left[1+\frac{b}{l_{s} \sigma_{s}} \tanh (l \sigma)\right]^{-1} \text {. }
$$

Equation (3) means that, when depositing a liquid layer on the substrate, the resulting PA signal drop depends not only on the liquid thermal properties but also on its thickness.

To include the effects of the liquid layer evaporation and contraction we have to incorporate the drop dynamics at the same time that, from the thermal properties point of view, the liquid layer is treated as an effective backing material to the substrate. The point is that as time elapses, both the liquid thickness as well as the substrate coverage change due to the evaporation and the droplet contraction. We are eventually left, at some given time, with a situation where only part of the substrate sensing area is covered by the liquid, as schematically shown in Fig. 4(b). In this case, the substrate may be viewed as being in thermal contact with an effective backing medium, part of which is air and part of which is the reduced liquid drop. For this effective backing consisting of two media (air and liquid) that are parallel, the thermal properties may be written as ${ }^{14}$

$$
\begin{aligned}
& k=\left(A_{L} k_{L}+A_{g} k_{g}\right) / A_{0}=x k_{L}+(1-x) k_{g}, \\
& \rho c=\left[A_{L}(\rho c)_{L}+A_{g}(\rho c)_{g}\right] / A_{0}=x(\rho c)_{L}+(1-x)(\rho c)_{g}
\end{aligned}
$$

and

$$
\begin{aligned}
& e=\sqrt{k \rho c}=e_{l}\{[x+(1-x) \lambda][x+(1-x) \delta]\}^{1 / 2}, \\
& \alpha=\frac{k}{\rho c}=\alpha_{L}[x+(1-x) \lambda] /[x+(1-x) \delta] .
\end{aligned}
$$

In Eq. (4) the subscript $L$ ( $g$ ) refers to the liquid (air), $A_{0}$ is the substrate sensing area, and $A_{L}$ and $A_{g}$ are the areas of the substrate in contact with the liquid and air, respectively. The parameter $x=\left(R(t) / R_{0}\right)^{2}$ is the fraction of the substrate sensing area which is in thermal contact with the liquid drop at time $t, \lambda=k_{g} / k_{L}$, and $\delta=(\rho c)_{g} /(\rho c)_{L}$. In Fig. 5 we show the dependence of the effective thermal diffusivity $\alpha$ and effusivity $e$, given by Eq. (4), as a function of sensing area coverage fraction for chloroform. The values of the physical parameters used were taken from the existing literature and are listed in Table I. Figure 5 shows that, starting from the liquid value, at the fully covered situation, the thermal diffusivity eventually reaches the air value when the droplet evaporation is completed at $x=0$.

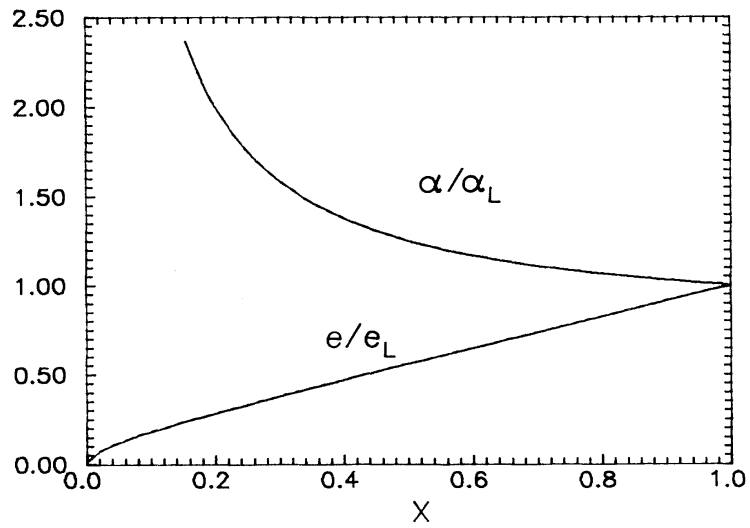

FIG. 5. Effective thermal diffusivity $\alpha / \alpha_{L}$ and thermal effusivity $e / e_{L}$ as a function of the substrate sensing area coverage for a chloroform droplet. 
TABLE I. Values of physical properties quoted in the literature (Ref. 16).

\begin{tabular}{llcccc}
\hline \multicolumn{1}{c}{ Substance } & $\begin{array}{c}\rho \\
\left(\mathrm{g} / \mathrm{cm}^{3}\right)\end{array}$ & $\begin{array}{c}c \\
(\mathrm{~J} / \mathrm{g} \mathrm{K})\end{array}$ & $\begin{array}{c}k \\
\left(\mathrm{~mW} / \mathrm{cm} \mathrm{K}^{2}\right)\end{array}$ & $\begin{array}{c}\alpha \\
\left(\mathrm{cm}^{2} / \mathrm{s}\right)\end{array}$ & $\begin{array}{c}e \\
\left(\mathrm{~W} \mathrm{~s}^{1 / 2} / \mathrm{cm}^{2} \mathrm{~K}\right)\end{array}$ \\
\hline Chloroform & 1.483 & 0.97 & 1.03 & 0.00072 & 0.0384 \\
Carbon tetrachloride & 1.594 & 0.86 & 1.03 & 0.00075 & 0.0376 \\
Trichloroethylene & 1.464 & 0.85 & 1.16 & 0.00073 & 0.0429 \\
Isopropanol & 0.785 & 2.5 & 1.4 & 0.00071 & 0.0525 \\
Aluminum & 2.71 & 0.90 & $2.28 \times 10^{3}$ & 0.93 & 2.36 \\
Air & 0.0012 & 1.003 & 0.2588 & 0.21 & 0.00056 \\
\hline \hline
\end{tabular}

The explicit time dependence of the drop radius and thickness is obtained from the droplet dynamics. Any attempt to analyze the self-spreading of a droplet on a plane surface encounters serious theoretical difficulties. ${ }^{9}$ In our case, the problem is further complicated by the fact that the droplet is evaporating. The droplet motion is controlled by forces at the solid-fluid contact line, and there is no simple answer to the question as to how these local molecular processes are represented within a macroscopic continuum theory. In static situations, the contact angle at the interface is the classical means of describing the balance of surface-tension forces there. The dynamic contact angle, which is the instantaneous slope angle at the moving edge, is the most natural extension of this concept.

One of the simplest situations dealing with the slow motion of a small, flat, viscous droplet is that for which lubrication theory ${ }^{8,9}$ is an adequate approximation. The physical situation is one for which viscous stresses and surface tension forces are predominant, inertial forces are unimportant, and the fluid is assumed to be always in a state of near equilibrium. The lubrication theory consists of a depth-averaged equation-of-mass conservation and a simplified form of the Navier-Stokes equations that is appropriate for a thin layer of viscous fluid. For small contact angles and small slip coefficients, the main conclusion of the lubrication theory ${ }^{8,9}$ is that the mean curvature of the droplet surface depends only on time and not on position. In other words, the surface curvature is spatially uniform during the droplet motion and a state of quasiequilibrium is established by the balance of internal and capillary pressures, both of which are functions of time only. Formally, this conclusion is expressed by requiring that

$$
\nabla^{2} h=-F(t),
$$

where $F(t)$ is an arbitrary function of time and $h(r, t)$ is droplet height (cf. Fig. 6). In other words, the boundary value problem to be solved is that of finding a solution to Poisson's equation within a moving boundary whose specification is part of the problem.

To apply the lubrication model to the problem of the radial contraction of a droplet that is evaporating, we consider the case of a circular droplet for which case the independent variables are the radius $r$ and time $t$. The moving contact line is $r=R(t)$, for which $h(R(t), t)=0$, and $R(t=0)=R_{0}$ is the initial drop radius. The solution to Eq. (5) which satisfies the condition $h(R(t), t)=0$ and is regular at the origin is

$$
h=A\left[1-\frac{r^{2}}{R^{2}}\right] \text {. }
$$

The arbitrary function $A$ is determined from the condition that the contact angle $\theta$ remains constant during the evaporation process, namely,

$$
-\left.\frac{d h}{d r}\right|_{r=R}=\tan \theta \simeq \theta .
$$

The result is $A=\theta R / 2$, so that droplet shape at time $t$ is given by

$$
h(r, t)=\frac{\theta R}{2}\left[1-\frac{r^{2}}{R^{2}}\right] .
$$

To find the equation of motion for $R(t)$ we consider a fairly flat droplet $(\theta$ small $)$ and assume that the rate at which heat is extracted from the droplet-free surface by the evaporation is equal to the heat conducted through the liquid film with a temperature drop $\Delta T$. Here, $\Delta T$ is the difference in temperature between the plane interface and the vapor at the liquid-free surface. Denoting by $L$ the latent heat of evaporation per unit mass, and by $\bar{h}=\theta R / 4$, the average droplet thickness, one can write

$$
L \frac{d M}{d t}=-\frac{k_{L} \Delta T}{\bar{h}} \pi R^{2} .
$$

Substituting in Eq. (9) the liquid mass $M$ by $\rho_{L} \pi \theta R^{3} / 4$, one finds

$$
R \dot{R}=-\frac{16 k_{L} \Delta T}{3 \rho_{L} L \theta^{2}} .
$$

Integrating Eq. (10) with the condition that $R(0)=R_{0}$,

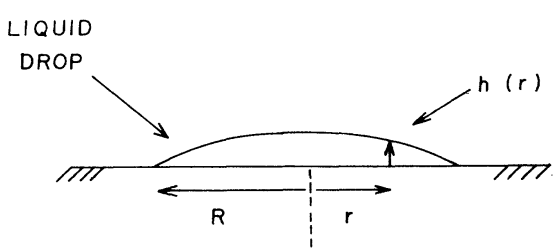

FIG. 6. Schematic droplet geometry. 
one finally gets

$$
X=\left[\frac{R(t)}{R_{0}}\right]^{2}=1-\frac{t}{\tau},
$$

where $\tau=3 \rho_{L} L R_{0}^{2} \theta^{2} / 32 k_{L} \Delta T$. Equation (11) means that, as the evaporation process takes place, the droplet area decreases linearly with time such that the full droplet evaporation occurs in a time $\tau$.

Equation (11) gives us the desired explicit timedependent expression for the parameter $X(t)$ representing the fraction of the substrate sensing area that is covered by the liquid film. The time-dependent expression for the liquid thickness $l(t)$ is obtained from the lubrication model by assuming that $l(t)=\bar{h}(t)=\bar{h}(0) \times(\bar{h}(t) /$ $\bar{h}(0))$. Noting that $\bar{h}(t)=\theta R(t) / 4$, denoting $\bar{h}(0)$ by $l_{0}$, the initial liquid film thickness, one finally gets

$$
l(t)=l_{0}\left(R(t) / R_{0}\right)=l_{0}\left(1-\frac{t}{\tau}\right)^{1 / 2},
$$

i.e., the liquid thickness decreases with the square root of $X(t)$.

The time-dependent effective backing model for PAdetected evaporation and contraction of a liquid droplet is completely defined by Eqs. (3), (4), (11), and (12). In Fig. 7 we show the predicted time evolution of the PA signal ratio for a chloroform droplet relative to the pure substrate signal [cf. Eq. (3)], using the values of the physical parameters listed in Table $I$ at a modulation frequency of $160 \mathrm{~Hz}$. To investigate the influence of the initial sample thickness on the PA signal we have chosen, as a reference sample, a droplet with an initial thickness of 30 $\mu \mathrm{m}$ and an evaporation time of $36 \mathrm{~s}$. The time evolution of this reference sample corresponds in Fig. 7 to curve $a$, whereas curves $b$ and $c$ represent the PA signal for droplets with initial thicknesses of 20 and $45 \mu \mathrm{m}$ and evaporation times of 16 and $81 \mathrm{~s}$, respectively. We note that, according to Eq. (11), the evaporation time scales with the square of the initial thickness. The overall behavior of the chloroform droplet evaporation simulation shown in

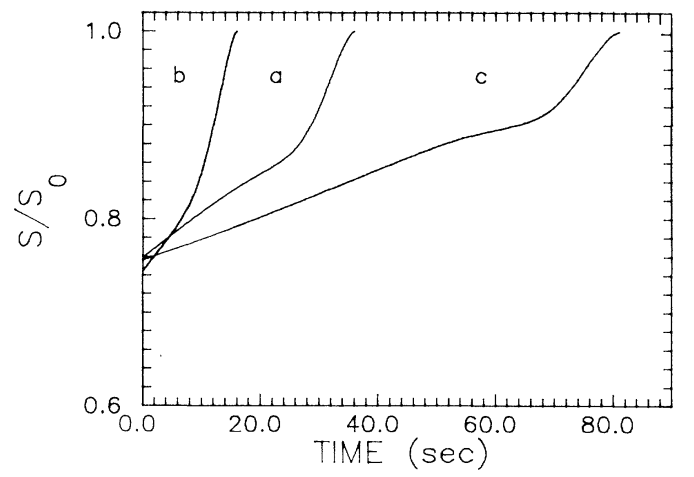

FIG. 7. Time dependence of a chloroform droplet PA signal $S$ relative to the pure substrate signal $S_{0}$, as predicted by the effective backing model, at a modulation frequency of $160 \mathrm{~Hz}$, for initial droplet thicknesses of (a) $30 \mu \mathrm{m}$, (b) $20 \mu \mathrm{m}$, and (c) 45 $\mu \mathrm{m}$.

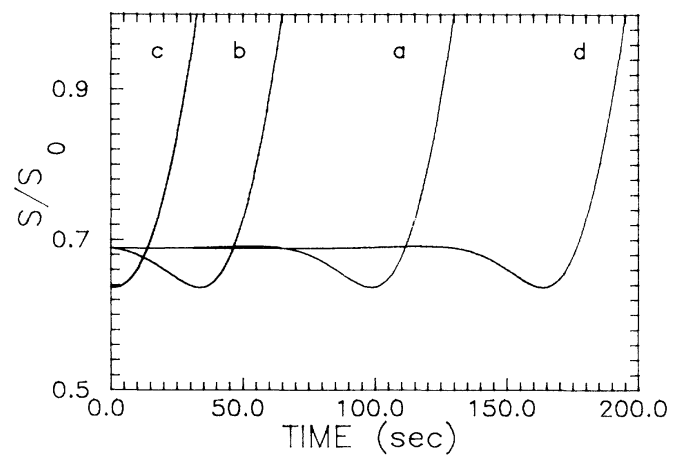

FIG. 8. Time dependence of an isopropanol droplet PA signal $S$ relative to the bare Al substrate signal $S_{0}$ as predicted by the effective backing model, at a modulation frequency of 160 $\mathrm{Hz}$, for initial droplet thicknesses of (a) $50 \mu \mathrm{m}$, (b) $25 \mu \mathrm{m}$, (c) $12.5 \mu \mathrm{m}$, and (d) $75 \mu \mathrm{m}$.

Fig. 7 seems to agree with the experimental observations mentioned in the previous section. In fact, Fig. 7 tells us that, just after the drop deposition, the PA signal ratio increases at a roughly constant rate, then it slows down for a few seconds and, finally, it rises quite rapidly to unity at the end of the evaporation process. The slowing down of the droplet contraction, which was visually observed during our experiments, is manifested in curve $c$ of Fig. 7 by the decrease of the signal derivative just before the fast rising region, at roughly $60 \mathrm{~s}$. For this sample, this slowing down point corresponds, according to Eq. (11), to $x=0.26$ which, in turn, entails that at this point the droplet radius is roughly half of the sensing area radius $R_{0}$, in agreement with our observations as previously mentioned. We note that $x \simeq 0.25$ corresponds to the point where the effective thermal diffusivity and effusivity, shown in Fig. 5, are both changing rapidly towards their air values.

The above model for the evaporating droplet explicitly neglects the effect of gravity on the droplet dynamics, which should be further discussed. Taking into account

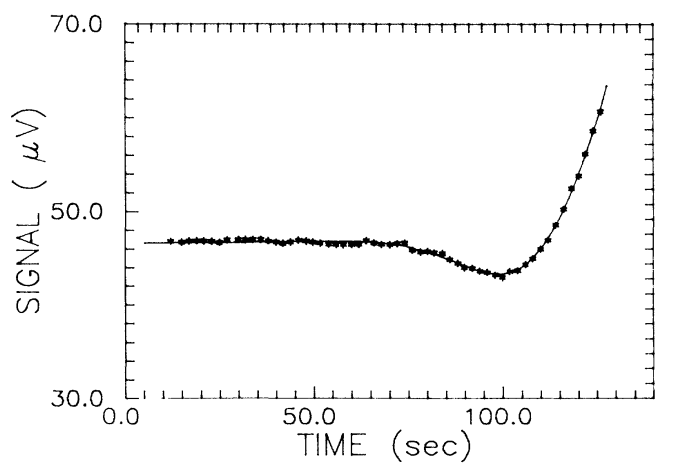

FIG. 9. Experimental data for the PA signal of an isopropanol droplet, recorded at a modulation frequency of $160 \mathrm{~Hz}$. The solid curve represents the result of the data fitting to the effective backing model. 
the gravity, the droplet curvature equation becomes

$$
\nabla^{2} h=-\frac{\rho g}{\gamma} h-F(t)
$$

instead of Eq. (5). Here, $\rho$ is the liquid density, $\gamma$ is the gas-liquid surface tension, and $g$ is the gravitational constant. The solution to Eq. (13) for a droplet of radius $R$ and contact angle $\theta$ subjected to the boundary conditions stated in Eq. (7) and the $h(R, t)=0$ is given by

$$
h(r, t)=R \theta\left[J_{0}(u z)-J_{0}(z)\right] / z J_{1}(z),
$$

where $J_{n}(x)$ is the $n$ th-order Bessel function $u=r / R$ and $z=R / \lambda c$. Here, $\lambda_{c}=(\gamma / \rho g)^{1 / 2}$ is the capillary length, which for most organic liquids is of the order of 1.5 to 2.0 $\mathrm{mm}$. Using the expansion of $J_{0}(u z)$ in terms of $J_{k}(z)$, namely,

$$
J_{0}(u z)=\sum_{k=1}^{\infty} \frac{\left(1-u^{2}\right)^{k}}{k !}\left[\frac{z}{2}\right]^{k} J_{k}(z),
$$

Eq. (14) reduces to

$$
h(r, t)=\frac{R \theta}{2} \sum_{k=1}^{\infty} \frac{\left(1-u^{2}\right)^{k}}{k !}\left[\frac{z}{2}\right]^{k-1} \frac{J_{k}(z)}{J_{1}(z)} .
$$

We note that in the case of small droplets with large capillary length (i.e., $\lambda_{c}$ very large), Eq. (15) reduces to Eq. (8) obtained neglecting the gravity effect. Using Eq. (15) the average droplet thickness $\bar{h}$ is written as

$$
\bar{h}=\frac{R \theta}{4} f(z)
$$

where $f(z)$ is given by

$$
f(z)=2 \sum_{m=0}^{\infty} \frac{1}{(m+2) !}\left[\frac{z}{2}\right]^{m} \frac{J_{m+1}(z)}{J_{1}(z)} .
$$

For $z \leq 3.0$, which is a reasonable range of values of $z$ for organic solvent droplets with initial radius of the order of $4 \mathrm{~mm}, f(z)$ can be analytically represented, within an error of $0.36 \%$ by the following expression:

$$
f(z)=1+0.017093 z \exp (0.93604 z) .
$$

For solvent droplets with an initial radius less than $4 \mathrm{~mm}$, the initial value of $z$, denoted by $z_{0}$, is typically of the order of 2.4. For this value of $z$, Eq. (18) is equal to 1.39, suggesting a $39 \%$ correction in the previous expression used for the average droplet thickness. However, the point to be noted is that, at the initial times, the liquid droplets are thermally thick [i.e., $l \sigma \gg 1$ in Eq. (3)] so that the signal ratio is roughly independent of $\bar{h}$. Fur-

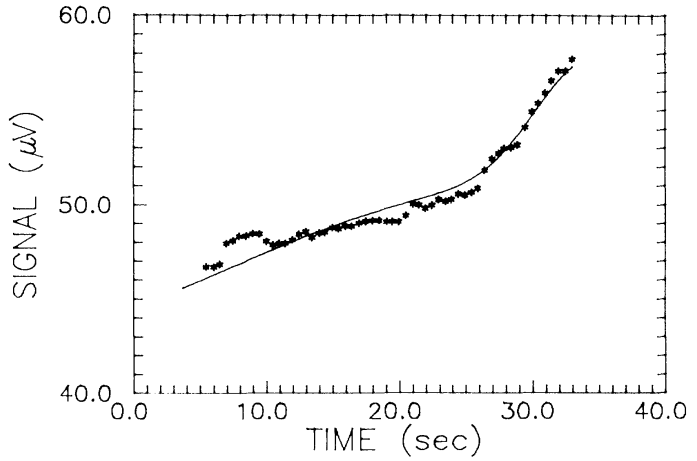

FIG. 10. Experimental data for the PA signal of a chloroform droplet recorded at a modulation frequency of $160 \mathrm{~Hz}$. The solid curve represents the result of the data fitting to the effective backing model.

thermore, it follows from Fig. 5 that the thermal diffusivity and effusivity change very little from their initial bare values until the initial radius has receeded to 0.84 of its $t=0$ value. This means that up to $R / R_{0}=0.84$ the signal ratio is roughly insensitive to $\bar{h}$. Writing $z$ as $z=z_{0}\left(R / R_{0}\right)$, and using $z_{0}=2.4$, the correction in $\bar{h}$, as given by Eq. (18), when $R / R_{0}=0.84$ (or, $z=2.0$ ) amounts to $22 \%$. As the droplet further recedes, $z$ gets smaller and the corrections to $\bar{h}=R \theta / 4$ become increasingly negligible. These calculations suggest that the gravity effect amounts actually to a correction of at most $22 \%$ to $\bar{h}$. This means that the above hydrodynamic model is, at least qualitatively, adequate for describing the PA-detected droplet evaporation.

So far we have discussed the case where the droplet evaporation is simultaneously followed by the droplet contraction. However, there are cases, like that of isopropanol droplets, that the evaporation takes place without a substantial change in the droplet radius. In this case of constant area evaporation, the only parameter in Eq. (3) that is changing with time is the liquid thickness $l(t)$. For such a situation, assuming that the heat flux conducted through the liquid layer is equal to $H \Delta T$, where $H$ is a heat transfer coefficient, the solution to the energy balance equation [cf., Eq. (10)] leads to

$$
l(t)=l_{0}\left(1-\frac{t}{\tau}\right)
$$

where $\tau=\rho L l_{0} / H \Delta T$. In other words, the liquid thickness decreases linearly with time for the case of constant area evaporation. We also note that the evaporation time

TABLE II. Values of the physical parameter obtained from the data fitting to the theoretical model.

\begin{tabular}{lcccc}
\hline \multicolumn{1}{c}{ Substance } & $\begin{array}{c}\tau \\
(\mathrm{sec})\end{array}$ & $\begin{array}{c}e \\
\left(\mathrm{~W} \mathrm{~s}^{1 / 2} / \mathrm{cm}^{2} \mathrm{~K}\right)\end{array}$ & $\begin{array}{c}l_{0} \\
(\mu \mathrm{m})\end{array}$ & $\begin{array}{c}\theta \\
(\mathrm{deg})\end{array}$ \\
\hline Chloroform & $35.60 \pm 1.36$ & $0.0353 \pm 0.0031$ & $32 \pm 1$ & $1.62^{\circ} \pm 0.21^{\circ}$ \\
Carbon tetrachloride & $63.24 \pm 0.83$ & $0.0354 \pm 0.0038$ & $30.7 \pm 2$ & $1.56^{\circ} \pm 0.31^{\circ}$ \\
Trichloroethylene & $73.50 \pm 6.85$ & $0.0396 \pm 0.0036$ & $30.1 \pm 0.5$ & $1.54^{\circ} \pm 0.18^{\circ}$ \\
Isopropanol & $127.73 \pm 5.86$ & $0.0502 \pm 0.0072$ & $51.3 \pm 3$ & $1.80^{\circ} \pm 0.32^{\circ}$ \\
\hline \hline
\end{tabular}


constant $\tau$ scales linearly with the liquid layer initial thickness $l_{0}$. This is consistent with the well known result ${ }^{15}$ that the mass loss due to evaporation from a flat surface varies linearly with time. Using Eq. (19) we have calculated the time-dependent PA or the case of isopropanol, for several values of the initial thickness $l_{0}$, at a modulation frequency of $160 \mathrm{~Hz}$. The results are shown in Fig. 8 in which curve $a$, regarded as our reference sample, corresponds to an isopropanol droplet having an initial thickness of $50 \mu \mathrm{m}$ and an evaporation time of $130 \mathrm{~s}$, whereas curves $b, c$, and $d$ refer to droplets of $25,12.5$, and $75 \mu \mathrm{m}$ thicknesses, and evaporation times of $65,32.5$, and $195 \mathrm{~s}$, respectively. As in the previous case of solvent evaporation, the behavior of the constant area evaporation model seems quite similar to the experimental results for isopropanol shown in Fig. 3.

\section{RESULTS AND DISCUSSIONS}

In this section, we apply the above time-dependent effective backing model to our experimental data. In Figs. 9 and 10 we present the results of our data fitting to the above model, for the cases of isopropanol and chloroform, respectively. In doing so, we have lifted as fitting parameters, the evaporation time $\tau$, the initial droplet thickness $l_{0}$, and the liquid thermal effusivity. The results for the physical parameters obtained from the data fitting for the investigated materials are summarized in Table II. For each substance we have performed a series of at least four experimental runs. We also show in Table II the values of the contact angle $\theta$ estimated using Eqs. (7) and (8) for the initial droplet thickness, namely, $l_{0}=\theta R_{0} / 4$. For the solvent droplets the initial radii were roughly the same for all the solvents used and of the order of $0.45 \pm 0.05 \mathrm{~cm}$, whereas for the isopropanol the droplet radii were estimated to be of the order of $0.65 \pm 0.05 \mathrm{~cm}$. The good agreement between theory and experiment suggests that the effective backing model, incorporating the lubrication theory describing the drop dynamics, provides us with adequate description for the PA monitored droplet evaporation.

Finally, to further check what the main assumptions of our model are based upon, namely, that the drop evaporation occurs with a constant receding contact angle and that the time history of the evaporating drop radius is such that its area decreases linearly with time, we have performed a simple experiment of monitoring the evaporating drop mass as a function of time. For the case of a receding solvent drop, using Eq. (11) and noting that its mass scales with $R^{3}$, the predicted drop mass time evolution is

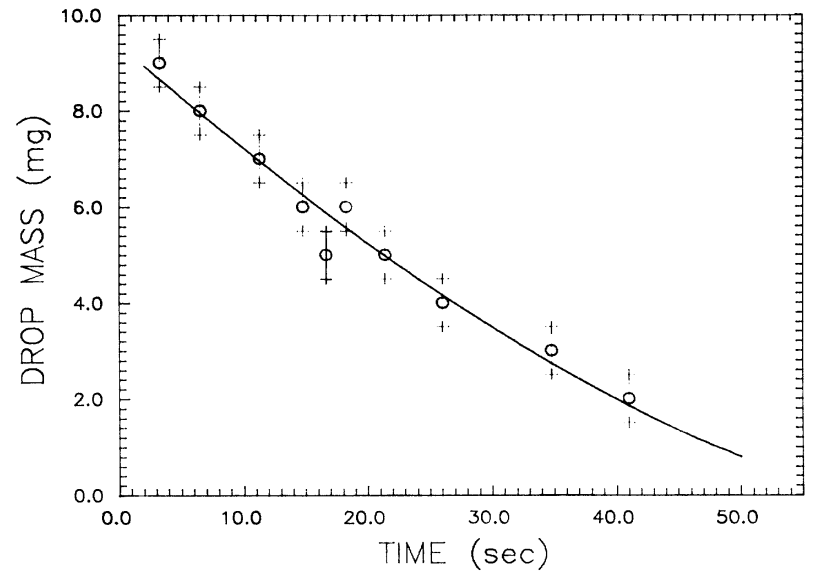

FIG. 11. Time dependence of the mass of one drop of carbon tetrachloride on a $12-\mu \mathrm{m}$-thick aluminum substrate. The solid line represents the result of the data fitting to Eq. (14) of the text.

$$
M(t)=M_{0}\left(1-\frac{t}{\tau}\right)^{3 / 2},
$$

where $M_{0}$ is the initial drop mass and $\tau$ is the evaporation time. The mass monitoring was performed by depositing one drop of the solvents on a $12-\mu \mathrm{m}$-thick aluminum substrate, using the same procedure as previously described, and measuring their masses as a function of time. The measurements were carried out using a weighing balance and the time base of an $X Y$ register for recording the time. For each material we have performed a series of nine experimental runs. The results we got for $\tau$ are in good agreement with the PA measured ones. As an example, we present in Fig. 11 the result we got for the mass time dependence for a $\mathrm{CCl}_{4}$ drop. The solid line in Fig. 11 represents the result of the data fitting to Eq. (20). This data fitting yielded a value of $\tau$ of $62.17 \pm 5.63 \mathrm{~s}$ which is quite close to the value of $63.24 \pm 0.83 \mathrm{~s}$ obtained from the PA measurements. We believe these complementary results provide further evidence supporting the proposed model for the real-time PA monitoring of an evaporating liquid drop. Furthermore, we hope that the suggested PA technique, as well as the other related photothermal techniques, may prove in the near future to become an important tool in the investigation of the many practical processes requiring the spreading of a liquid and a solid. Despite their importance, these processes are still poorly understood and a few sensitive techniques (fluorescence, EPR, etc.) can be applied specifically to the solidliquid interface.
${ }^{1}$ A. Rosencwaig, Photoacoustics and Photoacoustic Spectroscopy (Wiley, New York, 1980).

${ }^{2}$ H. Vargas and L. C. M. Miranda, Phys. Rep. 161, 43 (1988).

${ }^{3}$ G. Chen and E. Yeung, Anal. Chem. 60, 2258 (1988).

${ }^{4}$ J. A. Sell, D. M. Heffelfinger, P. L. G. Ventzeg, and R. M. Gilgenbach, J. Appl. Phys. 69, 1330 (1991).
${ }^{5}$ P. Poulet, D. Cahen, and S. Malkin, Biochim. Biophys. Acta 724, 433 (1983).

${ }^{6} \mathrm{P}$. Korpiun, R. Osiander, B. Büchner, and W. Herrmann, in Photoacoustics and Photothermal Phenomena, edited by $\mathrm{P}$. Hess and J. Pelzl (Springer-Verlag, Heidelberg, 1988), p. 192.

${ }^{7}$ J. Folkman and H. P. Greenspan, Biochim. Biophys. Acta 417, 
211 (1976).

${ }^{8}$ H. P. Greenspan, J. Fluid Mech. 84, 125 (1978).

${ }^{9}$ S. Rosenblat and S. H. Davis, in Frontiers in Fluid Mechanics, edited by S. H. Davis and J. L. Lumley (Springer-Verlag, Berlin, 1985), p. 171.

${ }^{10}$ A. Torres-Filho, L. F. Perondi, and L. C. M. Miranda, J. Appl. Polym. Sci. 35, 103 (1988).

${ }^{11}$ O. Pessoa, Jr., C. L. Cesar, N. A. Patel, H. Vargas, C. C. Ghizoni, and L. C. M. Miranda, J. Appl. Phys. 59, 1316
(1986).

${ }^{12}$ N. F. Leite, N. Cella, H. Vargas, and L. C. M. Miranda, J. Appl. Phys. 61, 3023 (1987).

${ }^{13}$ A. Rosencwaig and A. Gersho J. Appl. Phys. 47, 64 (1976).

${ }^{14}$ A. M. Mansanares, A. C. Bento, H. Vargas, N. F. Leite, and L. C. M. Miranda, Phys. Rev. B 42, 4477 (1990).

15J. P. Holman, Heat Transfer (McGraw-Hill, New York, 1976). ${ }^{16}$ Handbook of Chemistry and Physics (Chemical Rubber Co., Cleveland, 1972). 